\title{
On Bounds of Eigenvalues of Complex Sturm-Liouville Boundary Value Problems
}

\author{
Wenwen Jian and Huaqing Sun \\ Department of Mathematics, Shandong University at Weihai, Weihai, Shandong 264209, China \\ Correspondence should be addressed to Huaqing Sun; huaqingsun@wh.sdu.edu.cn
}

Received 25 March 2014; Accepted 1 May 2014; Published 12 May 2014

Academic Editor: Shurong Sun

Copyright (C) 2014 W. Jian and H. Sun. This is an open access article distributed under the Creative Commons Attribution License, which permits unrestricted use, distribution, and reproduction in any medium, provided the original work is properly cited.

The paper is concerned with eigenvalues of complex Sturm-Liouville boundary value problems. Lower bounds on the real parts of all eigenvalues are given in terms of the coefficients of the corresponding equation and the bound on the imaginary part of each eigenvalue is obtained in terms of the coefficients of this equation and the real part of the eigenvalue.

\section{Introduction}

Consider the regular complex Sturm-Liouville problem

$$
\tau y:=-y^{\prime \prime}+q y=\lambda w y
$$

in $L_{w}^{2}[0,1]$ associated with the Dirichlet boundary conditions

$$
y(0)=y(1)=0,
$$

where $q$ is a complex-valued function and $w$ is a real-valued function subjected to

$$
w(x)>0, \quad \text { a.e. } x \in[0,1], \quad q, w \in L^{1}[0,1] ;
$$

$\lambda$ is a spectral parameter, $L_{w}^{2}[0,1]$ is the weighted Hilbert space of all Lebesgue measurable complex-valued functions $f$ on $[0,1]$ satisfying $\int_{0}^{1} w|f|^{2}<\infty$ with the inner product $\langle f, g\rangle=\int_{0}^{1} w f \bar{g}$ and the norm $\|f\|=\langle f, f\rangle^{1 / 2}$, and $L^{1}[0,1]$ is the set of all Lebesgue measurable complex-valued functions $f$ on $[0,1]$ for which $\int_{0}^{1}|f|<\infty$.

Equation (1) is formally self-adjoint, if and only if $q$ is a real-valued function. Hence, (1) is formally non-self-adjoint, when the imaginary part of $q$ is nonzero. The boundary value problems associated with (1) with real coefficients have been deeply studied (cf., e.g., [1-5] and their references). Similar to regular self-adjoint boundary value problems, one can prove that (1) and (2) also have only countable eigenvalues without finite accumulation points using the spectral theory of compact operators in Hilbert spaces when (1) is formally non-self-adjoint. Unlike self-adjoint boundary value problems, (1) and (2) may have infinitely many nonreal eigenvalues, when the imaginary part of $q$ is nonzero (see [6, Theorem 1.1]). The asymptotic behavior of eigenvalues of boundary value problems associated with (1) has been studied (cf. [7-9]). Sufficient conditions were given in [10] for all eigenvalues of (1) with the periodic, antiperiodic, Dirichlet, or Neumann boundary conditions to be simple. For more related results for non-self-adjoint differential expressions, the reader is referred to [11-14] and the references cited therein.

In this paper, we are interested in the bounds on the eigenvalues of (1) and (2). In the case where (1) is formally self-adjoint, the bounds on eigenvalues of (1) and (2) were constructed in terms of the coefficients of (1), the coefficients of a comparing equation, and the eigenvalues of the comparing eigenvalue problem using the comparison theorem in [4]. The Rayleigh-Ritz method was used in [3] to obtain the bounds of eigenvalues of (1) and (2), when $q$ is positive and $w \equiv 1$. It is noted that the comparison theorem and the Rlayleigh-Ritz method are not applicable to boundary value problems (1) and (2), for the case where (1) is formally nonself-adjoint. Here, we obtain lower bounds on the real parts of all eigenvalues of (1) and (2) in terms of the coefficients of (1) 
and get the bound on the imaginary part of each eigenvalue in terms of the coefficients of (1) and the real part of the eigenvalue.

In the next section, we will present the main results of this paper.

\section{The Bounds on the Eigenvalues}

We denote by $\|\cdot\|_{C}$ the maximum norm of $C[0,1]$, which is the set of the continuous functions on $[0,1]$, by $\|\cdot\|_{1}$ the norm of the space $L^{1}[0,1]$ and by $\|\cdot\|_{2}$ the norm of the space $L^{2}[0,1]$. Let $q_{1}$ and $q_{2}$ be the real and imaginary parts of $q$, respectively; that is, $q_{1}=\operatorname{Re} q$ and $q_{2}=\operatorname{Im} q$. Then, $q=q_{1}+i q_{2}$, where $i=\sqrt{-1}$. For convenience, we set

$$
q_{k}^{-}:=-\min \left\{q_{k}, 0\right\}, \quad q_{k}^{+}:=\max \left\{q_{k}, 0\right\}, \quad k=1,2 .
$$

In addition, we denote

$$
S_{1}(\varepsilon):=\{x \in[0,1]: w(x)<\varepsilon\}, \quad m_{1}(\varepsilon):=\operatorname{mes} S_{1}(\varepsilon),
$$

$S_{2}(\varepsilon):=\left\{x \in[0,1]: w^{2}(x)<\varepsilon\right\}, \quad m_{2}(\varepsilon):=\operatorname{mes} S_{2}(\varepsilon)$.

Then the result below is one of the main results of the paper.

Theorem 1. If $\lambda$ is an eigenvalue of (1) and (2), then

$$
\operatorname{Re} \lambda \geq-\frac{8}{\varepsilon_{1}}\left(\left\|q_{1}^{+}\right\|_{1}+2\left\|q_{1}^{-}\right\|_{1}\right)\left\|q_{1}^{-}\right\|_{1}
$$

if $\lambda$ is an eigenvalue of (1) and (2), with $\operatorname{Re} \lambda \leq 0$, then

$$
|\operatorname{Im} \lambda| \leq \frac{8}{\varepsilon_{1}}\left\|q_{2}\right\|_{1}\left\|q_{1}^{-}\right\|_{1},
$$

where $\varepsilon_{1}>0$ satisfies $8\left\|q_{1}^{-}\right\|_{1}^{2} m_{1}\left(\varepsilon_{1}\right)<1$; and if $\lambda$ is an eigenvalue of (1) and (2), with $\operatorname{Re} \lambda>0$, then

$$
|\operatorname{Im} \lambda| \leq \frac{8}{\widetilde{\varepsilon}_{1}}\left\|q_{2}\right\|_{1}\left(\left\|q_{1}^{-}\right\|_{1}+\operatorname{Re} \lambda\|w\|_{1}\right),
$$

where $\widetilde{\varepsilon}_{1}>0$ satisfies $8\left(\left\|q_{1}^{-}\right\|_{1}+\operatorname{Re} \lambda\|w\|_{1}\right)^{2} m_{1}\left(\widetilde{\varepsilon}_{1}\right)<1$.

Proof. We first consider the case where $\lambda$ is an eigenvalue of (1) and (2), with $\operatorname{Re} \lambda \leq 0$. Let $\lambda$ be such an eigenvalue and let $\phi(x)$ be the corresponding eigenfunction with $\|\phi\|_{2}=1$. Then, $\phi(0)=\phi(1)=0$ and

$$
-\phi^{\prime \prime}+q \phi=\lambda w \phi .
$$

By multiplying both sides of (10) by $\bar{\phi}$ and integrating over the interval $[0,1]$, we have

$$
\int_{0}^{1}\left|\phi^{\prime}\right|^{2}+\int_{0}^{1} q|\phi|^{2}=\lambda \int_{0}^{1} w|\phi|^{2} .
$$

By separating the real and imaginary parts of both sides of (11), we get that

$$
\begin{gathered}
\operatorname{Re} \lambda \int_{0}^{1} w|\phi|^{2}=\int_{0}^{1}\left|\phi^{\prime}\right|^{2}+\int_{0}^{1} q_{1}|\phi|^{2}, \\
\operatorname{Im} \lambda \int_{0}^{1} w|\phi|^{2}=\int_{0}^{1} q_{2}|\phi|^{2} .
\end{gathered}
$$

From $\operatorname{Re} \lambda \leq 0$ and (12), it follows that

$$
\int_{0}^{1}\left|\phi^{\prime}\right|^{2} \leq \int_{0}^{1} q_{1}^{-}|\phi|^{2}
$$

Now, let $Q_{1}(x)=\int_{0}^{x} q_{1}^{-}(t) \mathrm{d} t$; then we have $\max _{x \in[0,1]}\left|Q_{1}(x)\right| \leq\left\|q_{1}^{-}\right\|_{1}$ and

$$
\begin{aligned}
\int_{0}^{1} q_{1}^{-}|\phi|^{2} & =\int_{0}^{1} Q_{1}^{\prime}|\phi|^{2}=-\int_{0}^{1} Q_{1}\left(\phi^{\prime} \bar{\phi}+\phi \overline{\phi^{\prime}}\right) \\
& =-2 \operatorname{Re} \int_{0}^{1} Q_{1} \phi^{\prime} \bar{\phi} \leq 2\left\|q_{1}^{-}\right\|_{1} \int_{0}^{1}\left|\phi^{\prime}\right||\bar{\phi}| \\
& \leq 2\left\|q_{1}^{-}\right\|_{1}\left\|\phi^{\prime}\right\|_{2} \leq 2\left\|q_{1}^{-}\right\|_{1}^{2}+\frac{1}{2}\left\|\phi^{\prime}\right\|_{2}^{2}
\end{aligned}
$$

by the Cauchy-Schwarz inequality. It can be obtained from (14) and (15) that

$$
\left\|\phi^{\prime}\right\|_{2}^{2} \leq 2\left\|q_{1}^{-}\right\|_{1}^{2}+\frac{1}{2}\left\|\phi^{\prime}\right\|_{2}^{2},
$$

and, hence,

$$
\left\|\phi^{\prime}\right\|_{2}^{2} \leq 4\left\|q_{1}^{-}\right\|_{1}^{2}, \quad \int_{0}^{1} q_{1}^{-}|\phi|^{2} \leq 4\left\|q_{1}^{-}\right\|_{1}^{2} .
$$

Similar to (15), we can get that

$$
\int_{0}^{1} q_{1}^{+}|\phi|^{2} \leq 2\left\|q_{1}^{+}\right\|_{1}\left\|\phi^{\prime}\right\|_{2}
$$

which, together with the first relation of (17), gives that

$$
\int_{0}^{1} q_{1}^{+}|\phi|^{2} \leq 4\left\|q_{1}^{+}\right\|_{1}\left\|q_{1}^{-}\right\|_{1} .
$$

In addition, from $\phi(x)=\int_{0}^{x} \phi^{\prime}(t) \mathrm{d} t$ and the Cauchy-Schwarz inequality, we have

$$
\begin{array}{r}
|\phi(x)|^{2}=\left|\int_{0}^{x} \phi^{\prime}(t) \mathrm{d} t\right|^{2} \leq x \int_{0}^{x}\left|\phi^{\prime}(t)\right|^{2} \mathrm{~d} t \leq \int_{0}^{1}\left|\phi^{\prime}\right|^{2}=\left\|\phi^{\prime}\right\|_{2}^{2}, \\
x \in[0,1],
\end{array}
$$

by which, together with (17), the definition of $m_{1}\left(\varepsilon_{1}\right)$ in (5), and $8\left\|q_{1}^{-}\right\|_{1}^{2} m_{1}\left(\varepsilon_{1}\right) \leq 1$, we get that

$$
\begin{aligned}
\int_{0}^{1} w|\phi|^{2} & \geq \int_{[0,1] \backslash S_{1}\left(\varepsilon_{1}\right)} w|\phi|^{2} \geq \varepsilon_{1} \int_{[0,1] \backslash S_{1}\left(\varepsilon_{1}\right)}|\phi|^{2} \\
& =\varepsilon_{1}\left(1-\int_{S_{1}\left(\varepsilon_{1}\right)}|\phi|^{2}\right) \\
& \geq \varepsilon_{1}\left(1-\left\|\phi^{\prime}\right\|_{2}^{2} m_{1}\left(\varepsilon_{1}\right)\right) \geq \frac{\varepsilon_{1}}{2} .
\end{aligned}
$$

Using (12), (17), (19), and (21), we can easily conclude that, for every eigenvalue $\lambda$ with $\operatorname{Re} \lambda \leq 0$,

$$
|\operatorname{Re} \lambda| \frac{\varepsilon_{1}}{2} \leq 4\left(\left\|q_{1}^{+}\right\|_{1}+2\left\|q_{1}^{-}\right\|_{1}\right)\left\|q_{1}^{-}\right\|_{1},
$$


which implies that (7) holds for all the eigenvalues of (1) and (2).

$$
\begin{aligned}
& \text { Let } Q_{2}(x)=\int_{0}^{x} q_{2}(t) \mathrm{d} t \text {. Then, from (17), } \\
& \qquad \int_{0}^{1} q_{2}|\phi|^{2} \leq 2\left\|q_{2}\right\|_{1}\left\|\phi^{\prime}\right\|_{2} \leq 4\left\|q_{2}\right\|_{1}\left\|q_{1}^{-}\right\|_{1},
\end{aligned}
$$

which, together with (13) and (21), implies that (8) holds for every eigenvalue $\lambda$ with $\operatorname{Re} \lambda \leq 0$.

Now, let $\lambda_{0}$ be an eigenvalue of (1) and (2), with $\operatorname{Re} \lambda_{0}>0$. Then, we consider the problem

$$
-y^{\prime \prime}+\left(q-\operatorname{Re} \lambda_{0} w\right) y=\lambda w y
$$

with the Dirichlet boundary conditions (2). It can be easily verified that $\lambda_{0}-\operatorname{Re} \lambda_{0}$ is an eigenvalue of (24) and (2). Clearly, $\operatorname{Re}\left(\lambda_{0}-\operatorname{Re} \lambda_{0}\right)=0$. Hence, by (8), there exists $\widetilde{\varepsilon}_{1}$ satisfying $8\left(\left\|q_{1}^{-}\right\|_{1}+\operatorname{Re} \lambda_{0}\|w\|_{1}\right)^{2} m_{1}\left(\widetilde{\varepsilon}_{1}\right)<1$ such that

$$
\left|\operatorname{Im} \lambda_{0}\right|=\left|\operatorname{Im}\left(\lambda_{0}-\operatorname{Re} \lambda_{0}\right)\right| \leq \frac{8}{\widetilde{\varepsilon}_{1}}\left\|q_{2}\right\|_{1}\left(\left\|q_{1}^{-}\right\|_{1}+\operatorname{Re} \lambda_{0}\|w\|_{1}\right) .
$$

This completes the proof.

The following corollary is a direct consequence of Theorem 1 .

Corollary 2. If $q_{1} \geq 0$, then all eigenvalues $\lambda$ of (1) and (2) satisfy that $\operatorname{Re} \lambda \geq 0$. Consequently, if there exists $q_{0} \in \mathbf{R}$ such that $q_{1} \geq q_{0} w$ on $[0,1]$, then all eigenvalues $\lambda$ of (1) and (2) satisfy that $\operatorname{Re} \lambda \geq q_{0}$.

If $w \in A C[0,1]$ and $w^{\prime} \in L^{2}[0,1]$, where $A C[0,1]$ denotes the set of functions which are locally absolutely continuous on $[0,1]$, then we have the following result.

Theorem 3. Assume that $w \in A C[0,1]$ and $w^{\prime} \in L^{2}[0,1]$. If $\lambda$ is an eigenvalue of (1) and (2), then

$$
\operatorname{Re} \lambda \geq-\frac{8}{\varepsilon_{2}}\left\|q_{1}^{-}\right\|_{1}^{2}\left[\|w\|_{C}\left(1+\left\|q_{1}\right\|_{1}\right)+\left\|w^{\prime}\right\|_{2}\right]
$$

if $\lambda$ is an eigenvalue of (1) and (2), with $\operatorname{Re} \lambda \leq 0$, then

$$
|\operatorname{Im} \lambda| \leq \frac{8}{\varepsilon_{2}}\left\|q_{1}^{-}\right\|_{1}^{2}\left(\|w\|_{C}\left\|q_{2}\right\|_{1}+\left\|w^{\prime}\right\|_{2}\right),
$$

where $\varepsilon_{2}>0$ satisfies $8\left\|q_{1}^{-}\right\|_{1}^{2} m_{2}\left(\varepsilon_{2}\right)<1$; and if $\lambda$ is an eigenvalue of (1) and (2), with $\operatorname{Re} \lambda>0$, then

$$
|\operatorname{Im} \lambda| \leq \frac{8}{\widetilde{\varepsilon}_{2}}\left(\left\|q_{1}^{-}\right\|_{1}+\operatorname{Re} \lambda\|w\|_{1}\right)^{2}\left(\|w\|_{C}\left\|q_{2}\right\|_{1}+\left\|w^{\prime}\right\|_{2}\right),
$$

where $\widetilde{\varepsilon}_{2}>0$ satisfies $8\left(\left\|q_{1}^{-}\right\|_{1}+\operatorname{Re} \lambda\|w\|_{1}\right)^{2} m_{2}\left(\widetilde{\varepsilon}_{2}\right)<1$.

Proof. Let $\lambda$ be an eigenvalue of (1) and (2) with $\operatorname{Re} \lambda \leq 0$ and let $\phi(x)$ be the corresponding eigenfunction with $\|\phi\|_{2}=1$. Then, $\phi(0)=\phi(1)=0$ and (10) holds. By multiplying both sides of (10) by $w \bar{\phi}$ and integrating over the interval $[0,1]$, we have

$$
\int_{0}^{1} w\left|\phi^{\prime}\right|^{2}+\int_{0}^{1} w^{\prime} \phi^{\prime} \bar{\phi}+\int_{0}^{1} w q|\phi|^{2}=\lambda \int_{0}^{1} w^{2}|\phi|^{2} .
$$

By separating the real and imaginary parts of both sides of (29), we get that

$$
\begin{gathered}
\operatorname{Re} \lambda \int_{0}^{1} w^{2}|\phi|^{2}=\int_{0}^{1} w\left|\phi^{\prime}\right|^{2}+\operatorname{Re}\left(\int_{0}^{1} w^{\prime} \phi^{\prime} \bar{\phi}\right)+\int_{0}^{1} w q_{1}|\phi|^{2}, \\
\operatorname{Im} \lambda \int_{0}^{1} w^{2}|\phi|^{2}=\operatorname{Im}\left(\int_{0}^{1} w^{\prime} \phi^{\prime} \bar{\phi}\right)+\int_{0}^{1} w q_{2}|\phi|^{2}
\end{gathered}
$$

On the other hand, it follows from (17) and (20) that

$$
\begin{gathered}
\int_{0}^{1} w\left|\phi^{\prime}\right|^{2} \leq\|w\|_{C}\left\|_{\phi^{\prime}}\right\|_{2}^{2} \leq 4\|w\|_{C}\left\|q_{1}^{-}\right\|_{1}^{2}, \\
\left.\left|\int_{0}^{1} w q_{1}\right| \phi\right|^{2} \mid \leq\|w\|_{C}\left\|\phi^{\prime}\right\|_{2}^{2}\left\|q_{1}\right\|_{1} \leq 4\|w\|_{C}\left\|q_{1}^{-}\right\|_{1}^{2}\left\|q_{1}\right\|_{1}, \\
\left|\int_{0}^{1} w^{\prime} \phi^{\prime} \bar{\phi}\right| \leq\left\|w^{\prime}\right\|_{2}\left\|\phi^{\prime}\right\|_{2}^{2} \leq 4\left\|w^{\prime}\right\|\left\|_{2}\right\| q_{1}^{-} \|_{1}^{2}, \\
\left.\left|\int_{0}^{1} w q_{2}\right| \phi\right|^{2} \mid \leq\|w\|_{C}\left\|q_{2}\right\|_{1}\left\|\phi^{\prime}\right\|_{2}^{2} \leq 4\|w\|_{C}\left\|q_{1}^{-}\right\|_{1}^{2}\left\|q_{2}\right\|_{1} .
\end{gathered}
$$

In addition, by (17), the definition of $m_{2}\left(\varepsilon_{2}\right)$ in (6), and $8\left\|q_{1}^{-}\right\|_{1}^{2} m_{2}\left(\varepsilon_{2}\right)<1$, it can be obtained that

$$
\begin{aligned}
\int_{0}^{1} w^{2}|\phi|^{2} & \geq \int_{[0,1] \backslash S_{2}\left(\varepsilon_{2}\right)} w^{2}|\phi|^{2} \geq \varepsilon_{2} \int_{[0,1] \backslash S_{2}\left(\varepsilon_{2}\right)}|\phi|^{2} \\
& =\varepsilon_{2}\left(1-\int_{S_{2}\left(\varepsilon_{2}\right)}|\phi|^{2}\right) \\
& \geq \varepsilon_{2}\left(1-4\left\|q_{1}^{-}\right\|_{1}^{2} m_{2}\left(\varepsilon_{2}\right)\right) \geq \frac{\varepsilon_{2}}{2},
\end{aligned}
$$

which, together with (30) and (32), implies that (26) holds for every eigenvalue $\lambda$ of (1) and (2), with $\operatorname{Re} \lambda \leq 0$, and, hence, (26) holds for all eigenvalues of (1) and (2). Furthermore, if $\lambda$ is an eigenvalue of (1) and (2), with $\operatorname{Re} \lambda \leq 0$, then (27) follows from (31)-(33). With a similar argument to that in the proof of Theorem 1, (28) can be proved. This completes the proof.

\section{Conflict of Interests}

The authors declare that there is no conflict of interests regarding the publication of this paper.

\section{Acknowledgments}

This research was supported by the NNSF of China (Grant 11101241), the NNSFs of Shandong Province (Grants ZR2011AQ002 and ZR2012AM002), and the Special Fund for Postdoctoral Innovative Programs of Shandong Province (Grant 201301010). 


\section{References}

[1] N. Dunford and J. T. Schwartz, Linear Operators, WileyInterscience, New York, NY, USA, 1963.

[2] E. Hille, Lectures on Ordinary Differential Equations, AddisonWesley, London, UK, 1969.

[3] C. O. Horgan and S. Nemat-Nasser, "Bounds on eigenvalues of Sturm-Liouville problems with discontinuous coefficients," Journal of Applied Mathematics and Physics, vol. 30, no. 1, pp. 77-86, 1979.

[4] J. R. McLaughlin, "Upper and lower bounds on eigenvalues of second-order Sturm-Liouville systems," Journal of Differential Equations, vol. 19, no. 2, pp. 201-213, 1975.

[5] A. Zettl, Sturm-Liouville Theory, vol. 121 of Mathematical Surveys and Monographs, American Mathematical Society, Providence, RI, USA, 2005.

[6] S. Albeverio, R. Hryniv, and Ya. Mykytyuk, "On spectra of nonself-adjoint Sturm-Liouville operators," Selecta Mathematica. New Series, vol. 13, no. 4, pp. 571-599, 2008.

[7] M. A. Naimark, Linear Differential Operators, George G. Harap, 1967.

[8] O. A. Veliev and M. T. Duman, "The spectral expansion for a nonself-adjoint Hill operator with a locally integrable potential," Journal of Mathematical Analysis and Applications, vol. 265, no. 1, pp. 76-90, 2002.

[9] B. Yilmaz and O. A. Veliev, "Asymptotic formulas for Dirichlet boundary value problems," Studia Scientiarum Mathematicarum Hungarica, vol. 42, no. 2, pp. 153-171, 2005.

[10] O. A. Veliev, "On the simplicity of the eigenvalues of the non-self-adjoint Mathieu-Hill operators," 2013, http://arxiv.org/abs/1301.1011.

[11] M. Chugunova and D. Pelinovsky, "Spectrum of a non-selfadjoint operator associated with the periodic heat equation," Journal of Mathematical Analysis and Applications, vol. 342, no. 2, pp. 970-988, 2008.

[12] R. Mennicken and M. Möller, Non-Self-Adjoint Boundary Eigenvalue Problems, Elsevier, San Diego, Calif, USA, 2003.

[13] D. Race, "A note on Dirichlet-type criteria for complex SturmLiouville expressions," Journal of Differential Equations, vol. 83, no. 2, pp. 336-347, 1990.

[14] H. Sun and J. Qi, "On classification of second-order differential equations with complex coefficients," Journal of Mathematical Analysis and Applications, vol. 372, no. 2, pp. 585-597, 2010. 


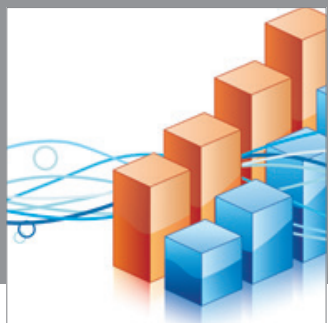

Advances in

Operations Research

mansans

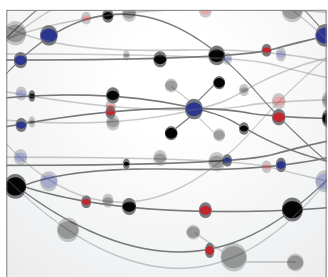

The Scientific World Journal
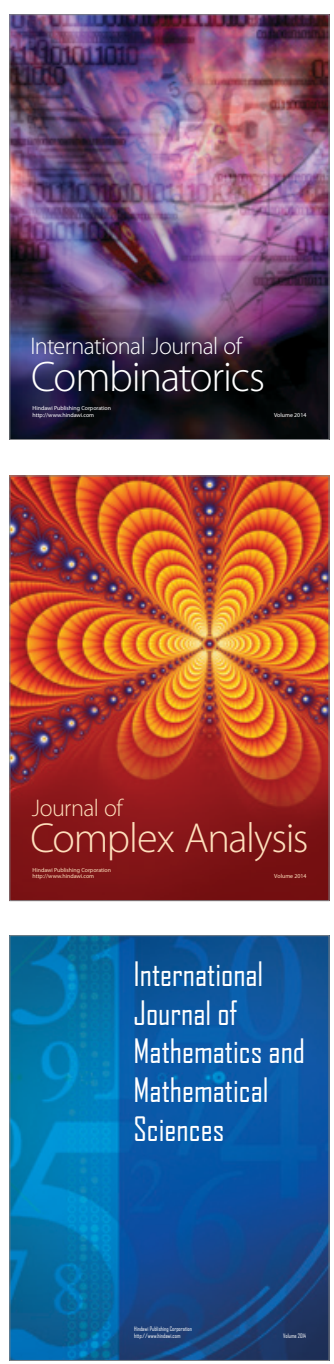
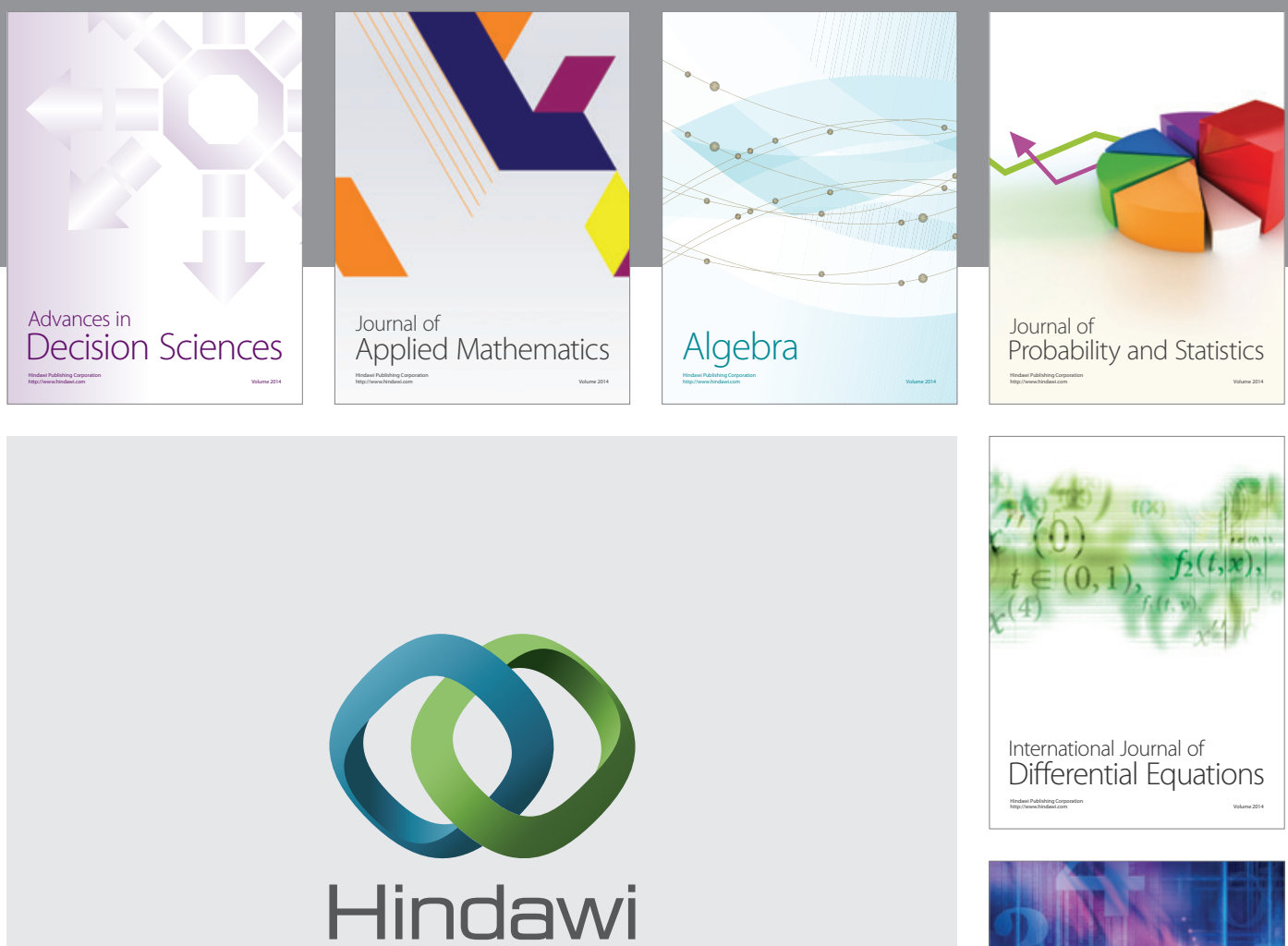

Submit your manuscripts at http://www.hindawi.com
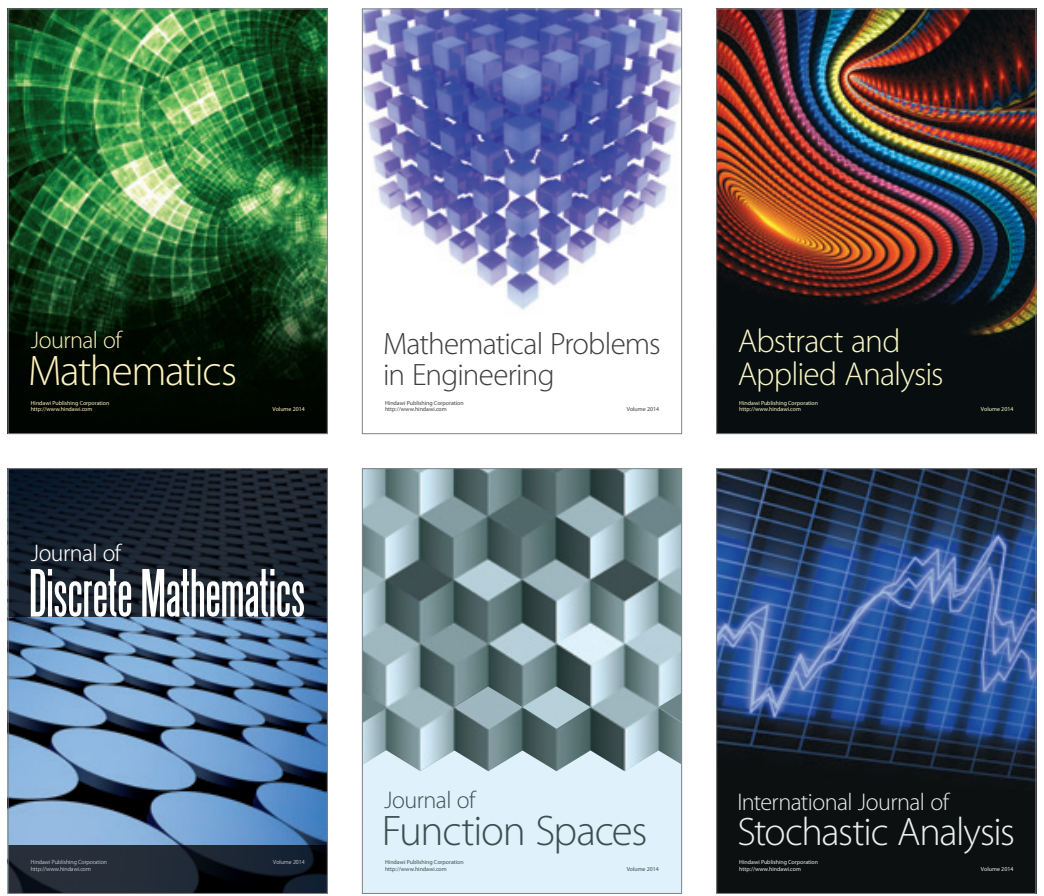

Journal of

Function Spaces

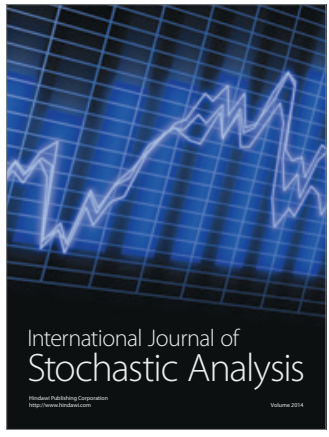

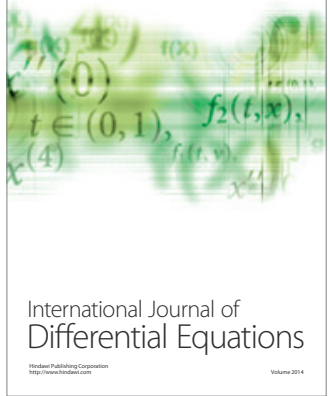
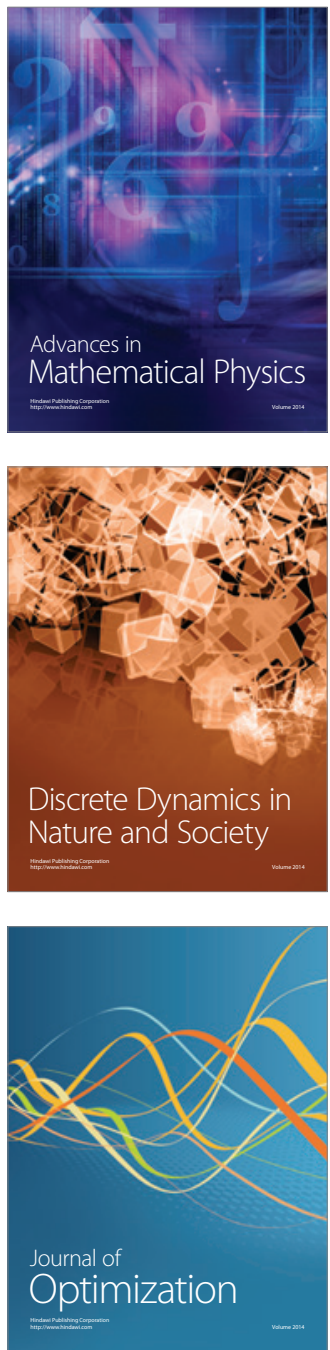